\title{
Analysis of Risk Factors for Lymph Node Metastases in Elderly Patients with Papillary Thyroid Micro-Carcinoma
}

This article was published in the following Dove Press journal: Cancer Management and Research

\author{
Gui-Ming Fu (D' \\ Zhao-Hui Wang' \\ Yi-Bo Chen' \\ Chun-Hua $\mathrm{Li}^{\prime}$ \\ Yue-Jia Zhang ${ }^{1,2}$ \\ Xiao-Jing $\mathrm{Li}^{1,2}$ \\ Quan-Xin Wan ${ }^{1,2}$
}

'Head and Neck Surgery Center, Sichuan Cancer Hospital and Institute, Sichuan Cancer Center, School of Medicine, University of Electronic Science and Technology of China, Chengdu 61004I, People's Republic of China; ${ }^{2}$ Department of Oncology, Chengdu Second People's Hospital, Chengdu 6I004I, People's Republic of China
Correspondence: Zhao-Hui Wang Email wangzh9I2@I63.com
Background: With guidance from the American Joint Committee on Cancer (AJCC) Cancer Staging Manual, 8th edition, we explored the characteristics of central lymph node metastasis (CLNM) of papillary thyroid micro-carcinoma (PTMC) in elderly patients $\geq 55$ years of age. Our goal was to provide references for establishing a lymph node dissection scheme in such patients.

Methods: We retrospectively analyzed the clinical data of thyroid cancer patients admitted to the Head and Neck Surgery Center of Sichuan Cancer Hospital, Chengdu, China, from January 2015 to September 2018. Then, we screened and analyzed eligible PTMC cases in strict accordance with our inclusion and exclusion criteria.

Results: The study included 107 patients, including 24 men and 83 women. Median age was $59.99 \pm 4.58$ years. The maximum diameter range of the cancer foci was $4-10 \mathrm{~mm}$, and the median was $7.59 \pm 1.78 \mathrm{~mm}$. Unilateral lobectomy had been performed in 32 cases, total thyroidectomy in 75 cases and lateral cervical lymph node dissection in 21 cases. There were 60 cases of CLNM (56.07\%) and 13 cases of lateral cervical lymph node metastasis $(12.10 \%)$. The sensitivity of preoperative ultrasound in predicting CLNM was 100\%, but its accuracy was only $50.47 \%$. Multivariate logistic regression analysis showed that multiple cancer foci (area under the curve $[\mathrm{AUC}]=0.632)$, extra-thyroidal expansion of cancer focus $(\mathrm{AUC}=0.721)$, and irregular nodules $(\mathrm{AUC}=0.603)$ were independent risk factors for CLNM of PTMC in elderly patients $(\mathrm{P}<0.05)$. Overall predictability for PTMC-CLNM was $80.30 \%$.

Conclusion: 1) Preoperative color Doppler ultrasound is not recommended as the basis for cervical lymph node dissection in PTMC patients. 2) For multiple cancer foci, irregular nodules, and elderly patients with PTMC extra-thyroidal expansion, we recommend a prophylactic central lymph node dissecting. 3) Nonsurgical observation of PTMC in elderly patients with low risk should be carefully selected.

Keywords: elderly patients, thyroid cancer, papillary carcinoma, micro-carcinoma, central lymph node metastasis

\section{Introduction}

In 1988, the World Health Organization defined thyroid cancer (TC) with a maximum tumor diameter of $10 \mathrm{~mm}$ as micro-carcinoma. Up to now, doctors in different countries and regions of the world still differ significantly on how to treat such diseases, including on whether to perform preventive lymph node dissection in the central region of the cancer. As the AJCC raised the age factor in the clinical staging of TC from 45 to 55 in 2017, it can be seen that the medical community 
tends to be more conservative in general on surgical treatment of TC. Based on clinical staging of TC in the AJCC Cancer Staging Manual, 8th edition, this study aimed to explore the characteristics of CLNM of PTMC in the elderly population ( $\geq 55$ years) so as to provide some references for developing clinical treatment plans for such patients.

\section{Methods}

\section{Patients}

We retrospectively analyzed the data of patients with PTMC admitted to the Head and Neck Surgery Center of Sichuan Cancer Hospital, Chengdu, China, from January 2015 to September 2018. Eligible patients were screened in strict accordance with our inclusion and exclusion criteria for relevant data statistics and analysis. Inclusion criteria were as follows: 1) Patient age was $\geq 55$ years. 2) All of the patients had been newly diagnosed and newly treated, with no previous history of thyroid surgery. 3) Postoperative pathological diagnosis based on paraffin-embedded sections was papillary carcinoma. 4) Benign nodules with or without pathology and $>10 \mathrm{~mm}$ in maximum diameter suggested by preoperative color ultrasound. 5) Patients' complete medical records were available. 6) All of the surgeons had $>10$ years' experience in thyroid surgery. 7) Patients had undergone surgical resection of glandular lobes and isthmus on the affected side, as well as lymph node dissection in the central region with or without lateral cervical lymph node dissection. Exclusion criteria were as follows: 1) Postoperative pathology indicated mixed carcinoma with papillary and other types of carcinoma. 2) Patient had refused lymph node dissection in the central area on the affected side. 3) There were multiple cancer lesions, with the sum of the maximum diameter $>10 \mathrm{~mm}$.

\section{Surgical Technique}

All of the patients had been operated on according to the "at least 2 " principle, namely lymph node dissection at least in the central area on the cancerous side. Total thyroidectomy, bilateral central-area lymph node dissection with or without lateral cervical lymph node dissection were performed on patients with preoperative cytological confirmation of bilateral multi-lobed carcinoma or lateral cervical lymph node metastasis. Those with papillary micro-carcinoma in one glandular lobe and benign nodules in the other glandular lobe (i.e., multiple nodules) with maximum diameter of
$>10 \mathrm{~mm}$ underwent concurrent total thyroidectomy and bilateral central lymph node dissection. Central-region lymph nodes were dissected in the following areas: upper boundary-lower hyoid margin, lower boundary-superior sternal fossa (upper margin of the unknown artery), and external-lateral margin of the carotid sheath and lower boundary-anterior vertebral fascia.

\section{Statistical Analysis}

We used SPSS software version 25.0 (SSPS, Inc., Chicago, Illinois, US) to statistically analyze all of the data. In our analysis of risk factors for lymph node metastasis in the central region, we performed single-factor analysis using $\chi^{2}$ test. We ran multivariate logistic-regression analysis on statistically significant positive univariate influencing factors, as well as univariate and multivariate receiver operating curve (ROC) analysis on the previously analyzed risk factors to predict lymph node metastasis in the central region.

\section{Results}

We screened a total of 712 PTMC cases. Of these, $107 \mathrm{met}$ the inclusion criteria, including 24 men and 83 women, with a male-to-female ratio of 1:3.46. Patients' age range was 55-74 years old, with a median of $59.99 \pm 4.58$ years. The maximum diameter range of the cancer lesion was 4-10 mm, median $7.59 \pm 1.78 \mathrm{~mm}$. There were 77 cases $(71.96 \%)$ with single-leaf single-focus, 9 (8.41\%) with single-leaf multi-focus and $21(19.63 \%)$ with multi-leaf multi-focus. Unilateral lobectomy was performed in 32 cases and total thyroidectomy in 75 cases and lateral cervical lymph node dissection in 21 cases. In terms of staging, $70.10 \%$ of cases were in stage T1 $(75 / 107)$, $22.42 \%$ in stage T3 $(24 / 107)$, and $7.48 \%$ in stage T4 (8/ 107). Postoperative pathology showed CLNM in 60 cases $(56.07 \%)$ and lateral cervical lymph node metastasis in 13 cases $(12.10 \%)$.

\section{Evaluation of Central Lymph Nodes by Color Doppler Ultrasound}

Preoperative ultrasound examination indicated 7 stage cN1a cases and 100 stage cN0 cases. Postoperative pathology confirmed 60 stage pN1a cases and 47 stage pN0 cases, which were significantly different. Predictive sensitivity, specificity, positive predictive value (PPV), and negative predictive value (NPV) were 1.00, 47.00\%, $11.67 \%$, and $1.00 \%$, respectively (Table 1 ). 
Table I Comparison of Preoperative Ultrasound Predictions of Central-Region Lymph Node Metastasis

\begin{tabular}{|c|c|c|c|c|c|}
\hline & \multirow[t]{2}{*}{$\mathbf{N}$} & \multicolumn{2}{|c|}{$\begin{array}{l}\text { Ultrasonic } \\
\text { Staging }\end{array}$} & \multirow[t]{2}{*}{ Total } & \multirow{2}{*}{$\begin{array}{l}\text { Sensitivity = } \\
1.00 \\
\text { Specificity = } \\
47.00 \%\end{array}$} \\
\hline & & $\mathrm{cNla}$ & cNO & & \\
\hline \multirow{2}{*}{$\begin{array}{l}\text { Pathological } \\
\text { stage }\end{array}$} & $\mathrm{pNIa}$ & 7 & 53 & 60 & $P P V=11.67 \%$ \\
\hline & pNo & 0 & 47 & 47 & $N P V=1.00$ \\
\hline Total & & 7 & 100 & 107 & $\begin{array}{l}\text { Matching rate = } \\
50.47 \%\end{array}$ \\
\hline
\end{tabular}

Abbreviations: PPV, positive predictive value; NPV, negative predictive value.

\section{Univariate Analysis}

In this study, single-factor analysis of 107 patients with PTMC age $\geq 55$ years showed that distribution, nodule morphology, calcification and extra-thyroidal expansion of cancer focus significantly influenced central-region lymph node metastasis $(P<0.05)$. However, patients' gender, thyroidstimulating hormone (TSH) levels, thyroglobulin (Tg) levels, nodular goiter, Hashimoto's thyroiditis, maximum diameter of cancer focus, nodular boundary, and nodular blood flow had no statistical significance on such metastasis (Table 2).

\section{Multivariate Logistic-Regression Analysis}

Factors that had been statistically significant in univariateanalysis results were further included in multivariate logistic-regression analysis; variables that might be clinically relevant but had been negative in univariate analysis were also included. We found that distribution, morphology and extra-thyroidal expansion of cancer focus were independent risk factors for CLNM $(P<0.05)$, while gender, TSH, $\mathrm{Tg}$, nodular goiters, Hashimoto's thyroiditis, nodular boundary, blood flow, calcification, and maximum diameter had no predictive significance (Table 3).

\section{ROC Curve Analysis}

We performed ROC curve analysis according to the independent risk factors obtained in our multivariate logistic regression analysis of CLNM, as discussed above, and we calculated areas under the curve (AUCs; Figures 1 and 2).

\section{Discussion}

\section{Disease Status of TC}

The incidence of TC has been on the rise globally over the past 30 years, which has been confirmed by most current studies. $^{1-3}$ According to International Agency for Research on Cancer (IARC) data for 2018, a total of 567,233 new cases (male:female $=1: 3.33$ ) and 41,071 deaths (male:female $=1: 1.64$ ) were reported in 185 countries around the world, respectively, accounting for 3.1\% and $0.4 \%$ of all new cancer cases and deaths. ${ }^{4}$ On the one hand, due to the great influence of medical ultrasound and cytological-puncture diagnosis, the proportion of PTMC in new cases of TC has increased significantly. According to the data, the overall incidence of PTMC in the United States has increased by $441 \%$ over the past 30 years, with average annual new cases accounting for about $49 \% .^{5-9}$ On the other hand, PTMC incidence in the elderly is significantly higher than that in the general adult population. Some studies have shown that the average annual growth rate of PTMC in patients $>65$ years old is 1.4 times that in adults $<65$ years old. ${ }^{8-10}$ These results indicate that we should pay sufficient attention to elderly PTMC patients. As the 8th edition of the AJCC Cancer Staging Manual raise PTC staging age from 45 to 55 years old, it further confirms this view.

\section{Therapeutic Controversies}

As we all know, diagnosis and treatment of PTMC have always been controversial, especially in elderly patients. In Japan's Kuma hospital, Ito et al defined the maximum diameter of thyroid cancer foci $\leq 1.0 \mathrm{~cm}$, no cervical, and distant lymph node metastasis, and cytological biopsy of thyroid cancer foci as non-highly malignant and no invasion of the trachea and recurrent laryngeal nerve as the judgment criteria for low-risk PTMC. After analyzing the data of 4023 patients, they concluded that immediate surgical treatment of all PTMC patients was more harmful than beneficial, so they suggested that low-risk PTMC patients should choose active observation. Among them, elderly PTMC patients over 60 years old were considered to be the most suitable group for observation. ${ }^{11,12}$ Conversely, Megwalu UC of the National Cancer Center (Plainview, New York, US) reviewed 2323 cases in which senile PTMC patients age $\geq 65$ received non-operative therapy. His data analysis shows that the overall 5-year survival rate was $23 \%$ and the surgery was $91 \%(P<0.0001)$, which suggests that surgery for such patients has a survival advantage, although more high-quality investigative studies are necessary. " "2015 American Thyroid Association Management Guidelines for Adult Patients with Thyroid Nodules and Differentiated Thyroid Cancer" suggested that suspicious malignant thyroid nodules with a maximum diameter of $<1.00 \mathrm{~cm}$ be followed up to $1.00 \mathrm{~cm}$ for cytological 
Table 2 Single-Factor Analysis of Central-Region Lymph Node Metastasis

\begin{tabular}{|c|c|c|c|c|c|}
\hline \multirow[t]{2}{*}{ Factors } & \multirow[t]{2}{*}{ Number $(n=107)$} & \multicolumn{2}{|c|}{ Central Lymph Node Metastasis (CLNM) } & \multirow[t]{2}{*}{$\chi^{2}$} & \multirow[t]{2}{*}{$\mathbf{P}$} \\
\hline & & Yes $(n=47)$ & No $(n=60)$ & & \\
\hline Gender & & & & 2.736 & 0.098 \\
\hline Male & 24 & 7 & 17 & & \\
\hline Female & 83 & 40 & 43 & & \\
\hline TSH levels & & & & 0.145 & 0.704 \\
\hline Normal & 86 & 37 & 49 & & \\
\hline Abnormal & 21 & 10 & 11 & & \\
\hline Tg levels & & & & - & 1.000 \\
\hline Normal & 99 & 44 & 55 & & \\
\hline Abnormal & 8 & 3 & 5 & & \\
\hline Nodular goiter & & & & 1.226 & 0.268 \\
\hline Yes & 55 & 27 & 28 & & \\
\hline No & 52 & 20 & 32 & & \\
\hline Hashimoto's thyroiditis & & & & 0.102 & 0.749 \\
\hline Yes & 22 & 9 & 13 & & \\
\hline No & 85 & 38 & 47 & & \\
\hline Distribution of carcinoma & & & & 9.992 & 0.005 \\
\hline Unilateral glandular lobes, single foci & 77 & 41 & 36 & & \\
\hline Unilateral glandular lobes, multiple foci & 9 & 1 & 8 & & \\
\hline Bilateral glandular lobes, multiple foci & 21 & 5 & 16 & & \\
\hline Maximum diameter & & & & 2.838 & 0.092 \\
\hline$\leq 5 \mathrm{~mm}$ & 12 & 8 & 4 & & \\
\hline $5 \mathrm{~mm}<x \leq 10 \mathrm{~mm}$ & 95 & 39 & 56 & & \\
\hline Boundary & & & & - & 0.329 \\
\hline Clear & 10 & 6 & 4 & & \\
\hline Unclear & 97 & 41 & 56 & & \\
\hline Echo & & & & - & 0.581 \\
\hline Low or no & 104 & 45 & 59 & & \\
\hline Strong or mixed & 3 & 2 & 1 & & \\
\hline Extra-thyroidal expansion & & & & 21.353 & $<0.01$ \\
\hline Yes & 63 & 16 & 47 & & \\
\hline No & 44 & 31 & 13 & & \\
\hline Calcification & & & & 5.541 & 0.019 \\
\hline Yes & 70 & 25 & 45 & & \\
\hline No & 37 & 22 & 15 & & \\
\hline Blood flow & & & & 0.439 & 0.508 \\
\hline Rich & 63 & 26 & 37 & & \\
\hline Not rich & 44 & 21 & 23 & & \\
\hline
\end{tabular}

Abbreviations: $\mathrm{TSH}$, thyroid-stimulating hormone; $\mathrm{Tg}$, thyroglobulin.

puncture and other treatments, but immediate surgical treatment is still recommended for high-risk patients. In this study, we performed surgical treatment on all PTMC patients with clear diagnoses, including stage T1 $(70.10 \%, 75 / 107)$, T3 $(22.42 \%, 24 / 107)$ and T4 $(7.48 \%, 8 / 107)$. Although the study sample size needs to be further expanded, we still believe that micro-carcinoma is not equal to early cancer. The percentage of differentiated tumor cells in elderly PTC patients is relatively higher than in youth and children, leading to shorter life expectancy. Choosing follow-up for middle-aged and elderly 
Table 3 Multivariate Logistic-Regression Analysis of Lymph Node Metastasis in the Central Region

\begin{tabular}{|c|c|c|c|c|c|c|}
\hline Factors & $\boldsymbol{\beta}$ & SE & Wald & $P$ & OR & 95\% Cl OR \\
\hline Gender & 0.958 & 0.671 & 2.036 & 0.154 & 2.606 & $0.699-9.714$ \\
\hline Distribution of carcinoma & 0.968 & 0.358 & 7.301 & 0.007 & 2.632 & $1.304-5.310$ \\
\hline Maximum diameter & -0.109 & 0.160 & 0.461 & 0.497 & 0.897 & $0.656-1.227$ \\
\hline Tumor formation pattern & 3.226 & 1.121 & 8.282 & 0.004 & 25.172 & $2.798-226.477$ \\
\hline Boundary & -1.752 & 1.218 & 2.070 & 0.150 & 0.173 & $0.016-1.886$ \\
\hline Extra-thyroidal expansion & 2.112 & 0.535 & 15.568 & 0.000 & 8.267 & $2.895-23.606$ \\
\hline Calcification & 1.082 & $0.58 \mathrm{I}$ & 3.468 & 0.063 & 2.950 & $0.945-9.209$ \\
\hline Blood flow & 0.230 & 0.533 & 0.186 & 0.666 & 1.258 & $0.443-3.577$ \\
\hline
\end{tabular}

Abbreviations: SE, Standard error; OR, odds ratio; $\mathrm{Cl}$, confidence interval.

PTMC patients may be feasible, but for those with longer life expectancy, early surgery can significantly reduce future progress of tumors may not only, but also reduced the forward of surgery as a result of basic diseases such as cardiovascular increase intolerance. Therefore, for PTMC patients age $\geq 55$ with good survival expectations, we believe surgical intervention is still necessary, which is also consistent with Shindo et al. ${ }^{13}$

\section{Risk Factors}

In the past, there have been many studies analyzing PTMC-CLNM, but few such reports address elderly patients with PTMC. Due to air interference in the tracheal cavity, it is relatively difficult to diagnose CLNM of the neck using ultrasound, which has a high false-negative rate. In this study, the accuracy of ultrasound in predicting CLNM was $50.47 \%$. Therefore, it is questionable whether dissection of such lymph nodes can be performed only by preoperative ultrasound. Chung et $\mathrm{al}^{14}$ found that in young PTMC patients, multiple cancer foci, enlarged nodules, extra-thyroidal expansion of cancer focus, and vascular invasion are independent risk factors for PTMC-CLNM and lateral cervical lymph node metastasis, but they did not clearly identify calcified nodules as an independent risk factor. By analyzing the data of 379 PTMC patients, Oh et al ${ }^{15}$ showed that the rate of lymph node metastasis in patients with calcified nodules was $17.8 \%$ higher than in patients whose nodules were not calcified; they concluded that calcified nodules were an important risk factor for PTMC cervical lymph node metastasis. Haugen et $\mathrm{al}^{16}$ have a similar view. In this study, the metastasis rates of the central cervical region and lateral cervical lymph nodes were $56.07 \%$ (60/107) and $12.10 \%$ (13/107), respectively. The $\chi^{2}$ test showed that distribution, nodular morphology, calcification and extra-thyroidal expansion of cancer focus were risk factors for central-region lymph nodes $(P<$

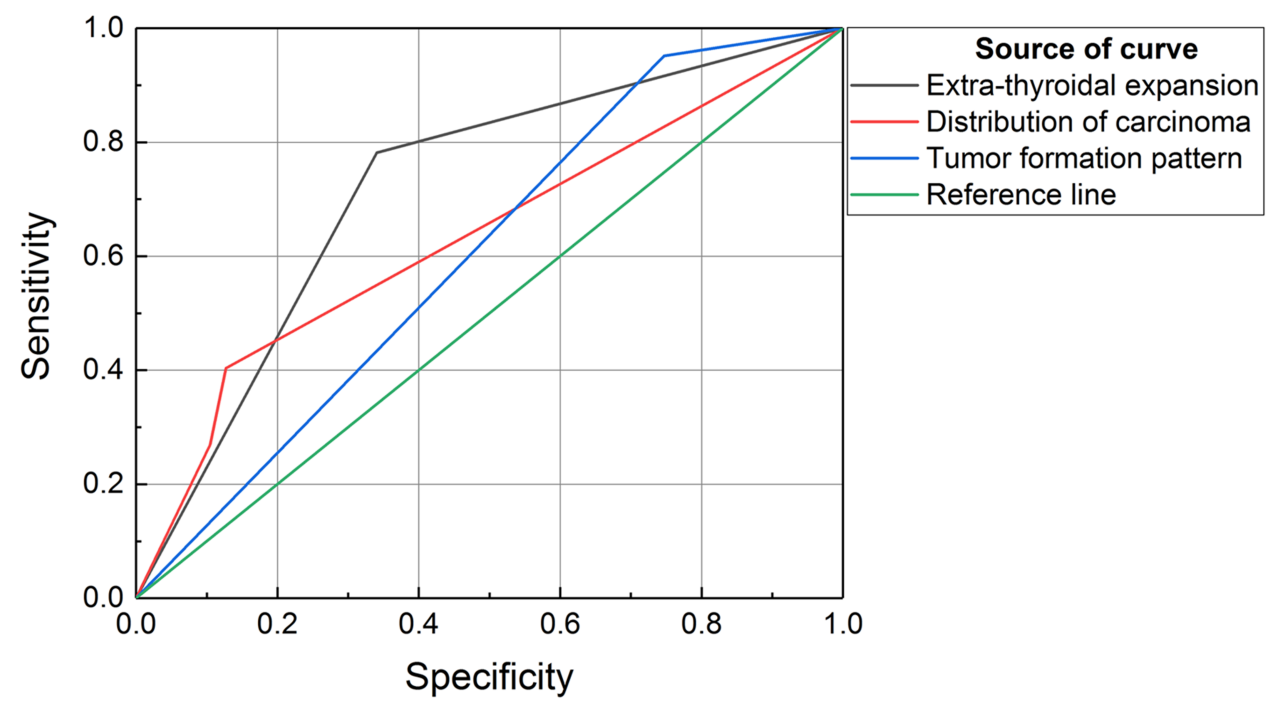

Figure I Areas under the curve (AUC). Distribution of carcinoma, AUC $=0.632$; extra-thyroidal expansion, $A U C=0.721$; tumor formation pattern, $A U C=0.603$. 


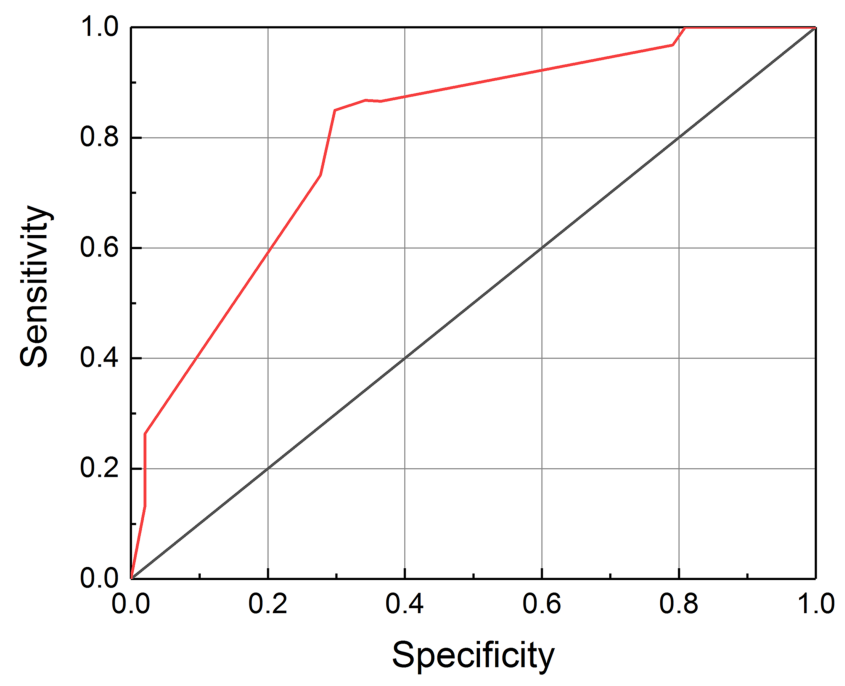

Figure 2 Multiple independent risk factors predicted lymph node metastasis in the central region. Areas under the curve $(A \cup C)=0.803$.

0.05), which was consistent with Liu et al. ${ }^{17}$ However, our multivariate logistic-regression analysis found that only distribution of cancer lesions $\left(\chi^{2}=7.301 ; P=0.007\right)$, nodule morphology $\left(\chi^{2}=8.282 ; P=0.004\right)$, and extrathyroidal expansion of cancer focus $\left(\chi^{2}=15.568\right.$; $P<0.001)$ were independent risk factors for such metastasis. The AUCs of these 3 factors were 0.632, 0.603 and 0.721 , respectively, and overall predictability was $80.30 \%$.

In summary, we believe that active follow-up and observation should be carefully selected for elderly patients with PTMC, especially for those with multiple cancer foci, extra-thyroidal expansion of cancer focus, and irregular morphology; preventive central-area lymph node dissection is also appropriate. Although we did not find nodular calcification, maximum tumor diameter, Hashimoto's thyroiditis, or other variables to be independent risk factors, we believe this result may have a certain relationship with the small sample size, which we will further expand in the future for related studies and supplements.

\section{Ethical Approval}

This study was approved by the Institutional Review Board of Sichuan Cancer Hospital and Institutional Ethics Committee and performed according to the ICH GCP principle.

\section{Informed Consent}

We obtained written informed consent from all of the individual participants included in the study.

\section{Acknowledgments}

All authors made substantial contributions to conception and design, acquisition of data, or analysis and interpretation of data; took part in drafting the article or revising it critically for important intellectual content; gave final approval of the version to be published; and agree to be accountable for all aspects of the work. We thank LetPub for its linguistic assistance during the preparation of this manuscript.

\section{Disclosure}

The authors report no conflicts of interest for this work.

\section{References}

1. Megwalu UC. Observation versus thyroidectomy for papillary thyroid microcarcinoma in the elderly. J Laryngol Otol. 2017;131 (2):173-176. doi:10.1017/S0022215116009762

2. Davies L, Welch HG. Current thyroid cancer trends in the United States. JAMA Otolaryngol Head Neck Surg. 2014;140(4):317-322. doi:10.1001/jamaoto.2014.1

3. Kilfoy BA, Zheng T, Holford TR, et al. International patterns and trends in thyroid cancer incidence, 1973-2002. Cancer Causes Control. 2009;20(5):525-531. doi:10.1007/s10552-008-9260-4

4. Bray F, Ferlay J, Soerjomataram I, et al. Global cancer statistics 2018: GLOBOCAN estimates of incidence and mortality worldwide for 36 cancers in 185 countries. CA Cancer J Clin. 2018;68 (6):394-424. doi:10.3322/caac.21492

5. Hughes DT, Haymart MR, Miller BS, et al. The most commonly occurring papillary thyroid cancer in the United States is now a microcarcinoma in a patient older than 45 years. Thyroid. 2011;21 (3):231-236. doi:10.1089/thy.2010.0137

6. Davies L, Welch HG. Increasing incidence of thyroid cancer in the United States, 1973-2002. JAMA. 2006;295(18):2164-2167. doi:10.1001/jama.295.18.2164

7. Kuo EJ, Goffredo P, Sosa JA, et al. Aggressive variants of papillary thyroid microcarcinoma are associated with extrathyroidal spread and lymph-node metastases: a population-level analysis. Thyroid. 2013;23(10):1305-1311. doi:10.1089/thy.2012.0563

8. Hay ID, Hutchinson ME, Gonzalez-Losada T, et al. Papillary thyroid microcarcinoma: a study of 900 cases observed in a 60 -year period. Surgery. 2008;144(6):980-987; discussion 987-988. doi:10.1016/j. surg.2008.08.035

9. Simard EP, Ward EM, Siegel R, et al. Cancers with increasing incidence trends in the United States: 1999 through 2008. CA Cancer J Clin. 2012;62(2):118-128. doi:10.3322/caac.20141

10. Cramer JD, Fu P, Harth KC, et al. Analysis of the rising incidence of thyroid cancer using the surveillance, epidemiology and end results national cancer data registry. Surgery. 2010;148(6):1147-1153. doi:10.1016/j.surg.2010.10.016

11. Ito Y, Miyauchi A, Kudo T, et al. Trends in the implementation of active surveillance for low-risk papillary thyroid microcarcinomas at Kuma Hospital: gradual increase and heterogeneity in the acceptance of this new management option. Thyroid. 2018;28(4):488-495. doi:10.1089/thy.2017.0448

12. Ito Y, Miyauchi A, Kihara M, et al. Patient age is significantly related to the progression of papillary microcarcinoma of the thyroid under observation. Thyroid. 2014;24(1):27-34. doi:10.1089/thy.2013.0367

13. Shindo M, Wu JC, Park EE, et al. The importance of central compartment elective lymph node excision in the staging and treatment of papillary thyroid cancer. Arch Otolaryngol Head Neck Surg. 2006;132(6):650-654. doi:10.1001/archotol.132.6.650 
14. Chung YS, Kim JY, Bae J-S, et al. Lateral lymph node metastasis in papillary thyroid carcinoma: results of therapeutic lymph node dissection. Thyroid. 2009;19(3):241-246. doi:10.1089/thy.2008.0244

15. Oh EM, Chung YS, Song WJ, et al. The pattern and significance of the calcifications of papillary thyroid microcarcinoma presented in preoperative neck ultrasonography. Ann Surg Treat Res. 2014;86 (3):115-121. doi:10.4174/astr.2014.86.3.115

16. Haugen BR, Alexander EK, Bible KC, et al. 2015 American Thyroid Association Management Guidelines for adult patients with thyroid nodules and differentiated thyroid cancer: the American Thyroid Association Guidelines task force on thyroid nodules and differentiated thyroid cancer. Thyroid. 2016;26(1):1-133. doi:10.1089/ thy.2015.0020
17. Liu L-S, Liang J, Li J-H, et al. The incidence and risk factors for central lymph node metastasis in cN0 papillary thyroid microcarcinoma: a meta-analysis. Eur Arch Otorhinolaryngol. 2017;274 (3):1327-1338. doi:10.1007/s00405-016-4302-0

\section{Publish your work in this journal}

Cancer Management and Research is an international, peer-reviewed open access journal focusing on cancer research and the optimal use of preventative and integrated treatment interventions to achieve improved outcomes, enhanced survival and quality of life for the cancer patient.
The manuscript management system is completely online and includes a very quick and fair peer-review system, which is all easy to use. Visit http://www.dovepress.com/testimonials.php to read real quotes from published authors. 